\title{
Adaptive Super-Twisting Algorithm-Based Nonsingular Terminal Sliding Mode Guidance Law
}

\author{
Fang Yang, ${ }^{1}$ Kuanqiao Zhang $\mathbb{D}^{2}{ }^{2}$ and Lei $Y u^{2}$ \\ ${ }^{1} X i$ 'an Aeronautical University, Xi'an 710077, China \\ ${ }^{2}$ Luoyang Electronic Equipment Test Center of China, Luoyang 471003, China \\ Correspondence should be addressed to Kuanqiao Zhang; zkuanqiao@163.com
}

Received 22 January 2020; Revised 28 May 2020; Accepted 11 June 2020; Published 4 July 2020

Academic Editor: Yu Wang

Copyright (c) 2020 Fang Yang et al. This is an open access article distributed under the Creative Commons Attribution License, which permits unrestricted use, distribution, and reproduction in any medium, provided the original work is properly cited.

A nonsingular fast terminal sliding mode guidance law with an impact angle constraint is proposed to solve the problem of missile guidance accuracy and impact angle constraint for maneuvering targets. Aiming at the singularity problem of the terminal sliding mode, a fast terminal sliding mode surface with finite-time convergence and impact angle constraint is designed based on fixedtime convergence and piecewise sliding mode theory. To weaken chattering and suppress interference, a second-order sliding mode supertwisting algorithm is improved. By designing the parameter adaptive law, an adaptive smooth supertwisting algorithm is designed. This algorithm can effectively weaken chattering without knowing the upper bound information of interference, and it converges faster. Based on the proposed adaptive supertwisting algorithm and the sliding mode surface, a guidance law with the impact angle constraint is designed. The global finite-time convergence of the guidance law is proved by constructing the Lyapunov function. The simulation results verify the effectiveness of the proposed guidance law, and compared with the existing terminal sliding mode guidance laws, the proposed guidance law has higher guidance precision and angle constraint accuracy.

\section{Introduction}

In modern warfare, many missiles (such as some antiship missiles, antitank missiles, and air defense missiles) need to hit the target with certain impact angles to increase the damage effectiveness of the warheads. Therefore, the impact angle constraint is a problem that needs to be considered in the design of the guidance law [1].

Sliding mode control is widely used in the design of the guidance law because of its invariability to interference in the sliding mode. In [2], the line-of-sight (LOS) angular velocity and impact angle constraint have been used as the sliding surface, and the sliding mode control is applied to design the guidance law with the impact angle constraint. In [3], the adaptive exponential approach law has been used to design the sliding mode guidance law, which increases the adaptability and dynamic performance of the guidance law. However, none of these methods has finite-time convergence. For the finite-time control problem, a finite-time convergence guidance law with the impact angle constraint is designed based on the terminal sliding mode control in [4-6]. In [7, 8], linear terms are added to the terminal sliding mode surface to further speed up the convergence of system states. But the negative exponential term of the state quantity in the terminal sliding mode control law will cause the singular problem. For the singular problem, a nonsingular terminal sliding surface is improved to avoid the singularity problem in [9-11], and the corresponding guidance law is designed. However, the proposed guidance laws cannot guarantee strictly finite-time convergence of the sliding mode surface, and there are nonconvergence factors, so the convergence rate will be reduced. In [12], the problem of nonstrict convergence of the sliding surface is studied, and a nonsingular terminal sliding surface with strictly finite-time convergence is proposed. However, the sliding surface function is not smooth, the system can only converge to a bounded region, and the specific range cannot be given.

For the disturbance problems such as target maneuvering and system disturbance, there are currently three methods for processing most documents: (1) designing the 
disturbance observer to estimate the disturbance in real time and online $[13,14]$; (2) designing the adaptive law to estimate the upper bound of disturbance $[15,16]$; and (3) using the robustness of the sliding mode control to resist interference. These methods need to introduce symbolic function terms which will make the control quantity discontinuous and easy to cause the chattering phenomenon. Most literature studies smooth the symbol terms to reduce chattering. However, at the same time, they also change the inherent structure of the sliding mode control and weaken the robustness of the sliding mode control system. For the chattering problem, a second-order sliding mode supertwisting algorithm is proposed in [17]. It has the advantages of simple form, avoiding chattering, and strong robustness. However, the control law of the supertwisting algorithm is not smooth, the parameter selection needs to know the upper boundary information of system disturbance, and the convergence speed is slow when the system states are far from the equilibrium point.

In order to solve the above problems, this paper improves an adaptive smooth supertwisting algorithm, which solves the problems of slow convergence speed and the unsmooth control law of the traditional supertwisting algorithm and greatly weakens the chattering problem of the sliding mode control. At the same time, the parameter adaptive law is designed against the disturbance without knowing the upper bound information of the disturbance. Based on the idea of fixed-time convergence and piecewise sliding surface, a nonsingular fast terminal sliding surface with the impact angle constraint is designed. A nonsingular fast terminal sliding mode guidance law with the impact angle constraint is proposed based on the adaptive supertwisting algorithm. The global finite-time convergence is proved by constructing the Lyapunov function. Finally, the effectiveness and superiority of the guidance law are verified by simulation experiments.

\section{Preparatory Knowledge}

2.1. Relative Dynamics between the Missile and the Target. In the inertial coordinate system, the relative motion relationship between the missile and the target is established as shown in Figure 1. $M$ and $T$ represent the missile and the target, respectively. $r$ is the relative distance between the missile and the target, and $q$ is the LOS angle. $v_{m}$ and $v_{t}$ are velocities of the missile and the target, respectively, and $\theta_{m}$ and $\theta_{t}$ are track angles of the missile and the target, respectively.

According to the relative motion relationship of the missile and the target, the relative motion equations of the missile and the target can be obtained as follows:

$$
\left\{\begin{array}{l}
\dot{r}=-v_{m} \cos \left(q-\theta_{m}\right)+v_{t} \cos \left(q-\theta_{t}\right), \\
r \dot{q}=v_{m} \sin \left(q-\theta_{m}\right)-v_{t} \sin \left(q-\theta_{t}\right), \\
a_{m}=v_{m} \dot{\theta}_{m} \\
a_{t}=v_{t} \dot{\theta}_{t} .
\end{array}\right.
$$

Differentiating $\dot{q}$ with respect to time gives

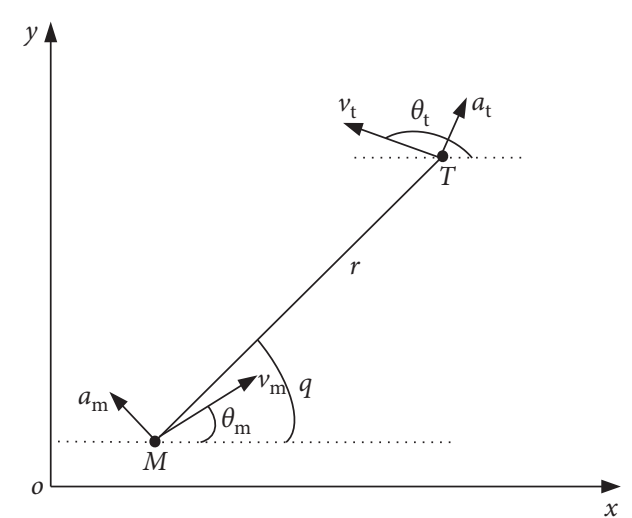

FIGURE 1: Relationship of missile-to-target motion.

$$
\ddot{q}=\frac{-2 \dot{r} \dot{q}}{r}-\frac{a_{m} \cos \left(q-\theta_{m}\right)}{r}+\frac{a_{t} \cos \left(q-\theta_{t}\right)}{r} .
$$

The impact angle is the angle between the missile and the target velocity vector at the time of guidance terminal, and the impact angle constraint problem can be transformed into the terminal LOS angle constraint problem [3-8]. Therefore, the state equation of the guidance system with the impact angle constraint can be obtained based on (2) as follows:

$$
\left\{\begin{array}{l}
\dot{x}_{1}=x_{2}, \\
\dot{x}_{2}=f_{1} x_{2}+f_{2} u+d,
\end{array}\right.
$$

with

$$
\left\{\begin{array}{l}
x_{1}=q-q_{d}, \\
x_{2}=\dot{q} \\
u=a_{m}, \\
f_{1}=-\left(\frac{2 \dot{r}}{r}\right), \\
f_{2}=-\left(\frac{1}{r}\right) . \\
d=\frac{\left(a_{m}-a_{m} \cos \left(q-\theta_{m}\right)+a_{t} \cos \left(q-\theta_{t}\right)\right)}{r},
\end{array}\right.
$$

where $q_{d}$ is the desired terminal LOS angle; $d$ can be regarded as the total disturbance of the system.

2.2. Related Lemma. For the convenience of analysis and proof, the following lemmas are introduced.

Lemma 1 (see [18]). Assume that there is a smooth function $V(\mathbf{x})$ defined on the neighborhood $\widehat{U} \subset U_{0} \subset \mathbf{R}^{n}$ of the origin, and $a_{1}>0$ and $0<b_{1}<1, \dot{V}(\mathbf{x})+a_{1} V^{b_{1}}(\mathbf{x}) \leq 0$; then, the origin of the system is finite-time stable, and the convergence time satisfies 


$$
T_{1} \leq \frac{V^{1-b_{1}}\left(\mathbf{x}_{0}\right)}{a_{1}\left(1-b_{1}\right)}
$$

Lemma 2 (see [19]). Assume that Lyapunov function $V(\mathbf{x})$ satisfies $\dot{V}(\mathbf{x}) \leq-a_{1} V^{b_{1}}(\mathbf{x})-a_{2} V(\mathbf{x})$, and $a_{1}>0, a_{2}>0$, and $0<b_{1}<1$; then, the system can converge to the origin in finite time, and the convergence time satisfies

$$
T_{2} \leq \frac{1}{a_{2}\left(1-b_{1}\right)} \ln \left(1+\frac{a_{2} V^{1-b_{1}}\left(\mathbf{x}_{0}\right)}{a_{1}}\right) .
$$

Lemma 3 (see $[20,21])$. For the nonlinear system $\dot{y}=-a_{1}|y|^{b_{1}} \operatorname{sgn}(y)-a_{2}|y|^{b_{2}} \operatorname{sgn}(y), \quad$ if $\quad a_{1}>0, \quad a_{2}>0$, $0<b_{1}<1$, and $b_{2}>1$, the system is stable in finite time, and the convergence time satisfies

$$
T_{3}<\frac{1}{a_{1}\left(1-b_{1}\right)}+\frac{1}{a_{2}\left(b_{2}-1\right)} .
$$

In addition, if the system has a small disturbance, that is, $\dot{y}=-a_{1}|y|^{b_{1}} \operatorname{sgn}(y)-a_{2}|y|^{b_{2}} \operatorname{sgn}(y)+\varsigma$ and $\varsigma$ is a small positive number, the system can converge to the neighborhood $\Omega=\left\{|y| \leq 2 \vartheta \mid a_{1} \vartheta^{b_{1}}+a_{2} \vartheta^{b_{2}}=\varsigma\right\}$ of the origin in finite time, and the convergence time satisfies

$$
T_{4}<\frac{1}{a_{1}\left(2^{b_{1}}-1\right)\left(1-b_{1}\right)}+\frac{1}{a_{2}\left(b_{2}-1\right)} .
$$

\section{Adaptive Fast Supertwisting Algorithm}

For the following first-order system,

$$
\dot{y}=u+\xi
$$

where $y$ is the system state, $u$ is the input, and $\xi$ is the disturbance, the supertwisting algorithm can be expressed as follows:

$$
\left\{\begin{array}{l}
u=-m_{1}|y|^{(1 / 2)} \operatorname{sgn}(y)+u_{1}, \\
\dot{u}_{1}=-m_{2} \operatorname{sgn}(y) .
\end{array}\right.
$$

The supertwisting algorithm can greatly reduce chattering and has strong robustness and high precision control performance [17]. However, the supertwisting algorithm has the following disadvantages: (1) the control law is a continuous function, but not a smooth function, which will affect the control performance; (2) the selection of control parameters needs to know the upper bound information of the system disturbances; and (3) when the system states are far from the equilibrium point, the convergence speed is slow. In view of the above shortcomings, this paper speeds up the convergence of the algorithm by adding linear terms to the algorithm. And the adaptive law does not need the information of the interference. The improved adaptive supertwisting algorithm can be expressed as follows:

$$
\left\{\begin{array}{l}
u=-m_{1} \varphi_{1}(y)+u_{1}, \\
\dot{u}_{1}=-m_{2} \varphi_{2}(y), \\
\varphi_{1}(y)=\frac{1}{\beta}|y|^{\beta} \operatorname{sgn}(y)+y, \\
\varphi_{2}(y)=y+\frac{|y|^{2 \beta-1} \operatorname{sgn}(y)}{\beta}+\left(\frac{1}{\beta}+1\right)|y|^{\beta} \operatorname{sgn}(y),
\end{array}\right.
$$

where $(1 / 2) \leq \beta<1$.

The parameter adaptive law is designed as follows:

$$
\left\{\begin{array}{l}
\dot{m}_{1}=(a+c) \varphi_{1}(y) \varphi_{2}(y) \operatorname{sgn}(|y|-\varepsilon), \\
m_{2}=b m_{1}, \\
m_{1}(0)>0
\end{array}\right.
$$

where $a>0, b>0, c>0$, and $\varepsilon>0$.

Substituting (11) into (9),

$$
\left\{\begin{array}{l}
\dot{y}=-m_{1} \varphi_{1}(y)+u_{1}+\xi \\
\dot{u}_{1}=-m_{2} \varphi_{2}(y)
\end{array}\right.
$$

Remark 1. It can be seen from (13) that when the system state is far away from the equilibrium point, the linear term $y$ in (11) will accelerate the convergence rate of the system. When the system is close to the equilibrium point, the nonlinear term $|y|^{\beta} \operatorname{sgn}(y)$ plays an important role in accelerating the convergence rate of the system. Therefore, compared with the traditional supertwisting algorithm, the improved adaptive supertwisting algorithm (11) has a faster convergence speed.

Remark 2. Due to the measurement noise of the system, the state of the system cannot reach the equilibrium point completely. In order to avoid the parameter increasing to infinity, the term $\operatorname{sgn}(|y|-\varepsilon)$ is added to the adaptive law to avoid the problem of overestimation [22].

Remark 3. It is obvious that $\varphi_{1}(s) \varphi_{2}(s) \geq 0$. So, it can be seen from (12) that when $|y|>\varepsilon, m_{1}$ and $m_{2}$ will gradually increase, making the system state to converge. When the system state converges to $|y|<\varepsilon, m_{1}$ and $m_{2}$ will decrease gradually. If $m_{1}$ and $m_{2}$ decrease to the point where the interference cannot be eliminated, the system state will deviate from $|y|<\varepsilon$. At this point, $m_{1}$ and $m_{2}$ will gradually increase under the effect of the adaptive law, making the system state converge to $|y|<\varepsilon$. Repeat the previous process; $m_{1}$ and $m_{2}$ will gradually decrease. Therefore, $m_{1}$ and $m_{2}$ are globally bounded.

For the total disturbance $\xi$ of the system, the following assumption can be made: 
Assumption 1. $\xi$ is bounded, $\xi=\xi_{1}+\xi_{2}, \quad \xi_{1}$ is nondifferentiable, and $\xi_{2}$ is differentiable; they satisfy

$$
\left\{\begin{array}{l}
\left|\xi_{1}\right| \leq K\left|\varphi_{1}(y)\right|, \\
\left|\xi_{2}\right| \leq L\left|\varphi_{2}(y)\right| .
\end{array}\right.
$$

Theorem 1. Under Assumption 1, the existence of $\bar{m}_{1}$ makes the system state converge in finite time when $m_{1} \geq \bar{m}_{1}$ and $m_{2}=b m_{1}$.

Proof. Define a new state vector as

$$
\mathbf{z}=\left[\begin{array}{l}
z_{1} \\
z_{2}
\end{array}\right]=\left[\begin{array}{c}
\varphi_{1}(y) \\
-m_{2} \int_{0}^{t} \varphi_{2}(y) d t+d_{2}
\end{array}\right] .
$$

From (14), it can be seen that the existence of $\rho_{1}(t)$ and $\rho_{2}(t)$ makes the following equation valid:

$$
\left\{\begin{array}{l}
\xi_{1}=\rho_{1} z_{1}, \quad\left|\rho_{1}\right| \leq K, \\
\dot{\xi}_{2}=\left(|y|^{\beta-1}+1\right) \rho_{2} z_{1}, \quad\left|\rho_{2}\right| \leq L .
\end{array}\right.
$$

Differentiating (15) with respect to time gives

$$
\begin{aligned}
\dot{z} & =\left[\begin{array}{l}
\dot{z}_{1} \\
\dot{z}_{2}
\end{array}\right] \\
& =\left(|y|^{\beta-1}+1\right)\left[\begin{array}{c}
-m_{1} z_{1}+\rho_{1} z_{1}+z_{2} \\
\rho_{2} z_{1}-m_{2} z_{1}
\end{array}\right] \\
& =\left(|y|^{\beta-1}+1\right) A z,
\end{aligned}
$$

with

$$
A=\left[\begin{array}{ll}
-m_{1}+\rho_{1} & 1 \\
-m_{2}+\rho_{2} & 0
\end{array}\right] .
$$

Define the following Lyapunov function:

$$
V_{1}=\mathbf{z}^{\mathrm{T}} \mathbf{P z}
$$

with

$$
\mathbf{P}=\left[\begin{array}{cc}
\left(\frac{a}{2}+b^{2}\right) & -b \\
-b & 1
\end{array}\right] .
$$

It is easy to prove that $\mathbf{P}$ is a positive definite matrix; then, $V_{1}$ is unbounded radially, i.e.,

$$
\lambda_{\text {min }}(\mathbf{P})\|\mathbf{z}\|^{2} \leq V_{1} \leq \lambda_{\max }(\mathbf{P})\|\mathbf{z}\|^{2} .
$$

Differentiating $V_{1}$ with respect to time gives

$$
\begin{aligned}
\dot{V}_{1} & =\left(|y|^{\beta-1}+1\right) \mathbf{z}^{\mathrm{T}}\left(\mathbf{A}^{\mathrm{T}} \mathbf{P}+\mathbf{P A}\right) \mathbf{z} \\
& =-\left(|y|^{\beta-1}+1\right) \mathbf{z}^{\mathrm{T}} \mathbf{Q} \mathbf{z},
\end{aligned}
$$

with

$$
\begin{aligned}
\mathbf{Q}= & {\left[\begin{array}{ll}
Q_{1} & Q_{2} \\
Q_{2} & 2 b
\end{array}\right], } \\
& \left\{\begin{array}{l}
Q_{1}=a m_{1}+2 b\left(b m_{1}-m_{2}\right)-\rho_{1}\left(a+2 b^{2}\right)+2 b \rho_{2}, \\
Q_{2}=m_{2}-b m_{1}-\left(\frac{a}{2}+b^{2}\right)+b \rho_{1}-\rho_{2} .
\end{array}\right.
\end{aligned}
$$

If we define

$$
\left\{\begin{array}{l}
\bar{m}_{1}=\frac{-\rho_{1}\left(a+2 b^{2}\right)+2 b \rho_{2}}{a}+\frac{\left(b \rho_{1}-\rho_{2}-(a / 2)-b^{2}\right)^{2}}{2 a b}, \\
m_{1} \geq \bar{m}_{1}, m_{2}=b m_{1},
\end{array}\right.
$$

it can be proved that $\mathbf{Q}$ is a positive definite matrix, and $\lambda_{\min }(\mathbf{Q}) \geq b$.

According to (21), we can get

$$
|y| \leq\|\mathbf{z}\| \leq\left(\frac{V_{1}}{\lambda_{\min }(\mathbf{P})}\right)^{(1 / 2)},
$$

$$
\begin{aligned}
\dot{V}_{1} & \leq-\left(\left(\frac{V_{1}}{\lambda_{\min }(\mathbf{P})}\right)^{(1-\beta / 2)}+1\right) \lambda_{\min }(\mathbf{Q})\|\mathbf{z}\|^{2} \\
& \leq-\frac{b \lambda_{\min }^{(1-\beta / 2)}(\mathbf{P}) V_{1}^{(1+\beta / 2)}}{\lambda_{\max }(\mathbf{P})}-\frac{b}{\lambda_{\max }(\mathbf{P})} V_{1} .
\end{aligned}
$$

According to Lemma 2, $\mathbf{z}$ is finite-time convergent. The proof is complete.

Theorem 2. Under the control law (11) and the control parameters which satisfy (12), the system state can converge to $|y| \leq \varepsilon$ in finite time.

Proof. According to (12), $m_{2}=b m_{1}$. If $m_{1} \geq \bar{m}_{1}$, then from Theorem 1, the system is finite-time convergent. If $m_{1}<\bar{m}_{1}$, define the Lyapunov function as

$$
V_{2}=\underbrace{\mathbf{z}^{\mathrm{T}} \mathbf{P} \mathbf{z}}_{V_{\mathbf{z}}}+\underbrace{\frac{\tilde{m}_{1}^{2}}{2}}_{V_{m 1}},
$$

where $\widetilde{m}_{1}=m_{1}-\bar{m}_{1}$.

Differentiating $V_{2}$ with respect to time gives

$$
\begin{aligned}
\dot{V}_{2}= & \left(|y|^{\beta-1}+1\right) \mathbf{z}^{\mathrm{T}}\left(\mathbf{A}^{\mathrm{T}} \mathbf{P}+\mathbf{P A}\right) \mathbf{z}+\dot{m}_{1} \widetilde{m}_{1} \\
= & \underbrace{\left(|y|^{\beta-1}+1\right) \mathbf{z}^{\mathrm{T}}\left(\bar{A}^{\mathrm{T}} \mathbf{P}+\mathbf{P} \bar{A}\right) \mathbf{z}}_{\dot{V}_{z}} \\
& +\underbrace{\left(|y|^{\beta-1}+1\right) \mathbf{z}^{\mathrm{T}}\left(\tilde{A}^{\mathrm{T}} \mathbf{P}+\mathbf{P} \tilde{A}\right) \mathbf{z}+\dot{m}_{1} \widetilde{m}_{1},}_{\dot{V}_{m 1}}
\end{aligned}
$$

with 


$$
\begin{aligned}
& \bar{A}=\left[\begin{array}{cc}
-\bar{m}_{1}+\rho_{1} & 1 \\
-b \bar{m}_{1}+\rho_{2} & 0
\end{array}\right], \\
& \widetilde{A}=\mathbf{A}-\bar{A}=\left[\begin{array}{cc}
-\widetilde{m}_{1} & 0 \\
-b \widetilde{m}_{1} & 0
\end{array}\right] \text {. }
\end{aligned}
$$

According to (26), we can get

$$
\dot{V}_{\mathbf{z}} \leq-\frac{b \lambda_{\min }^{(1-\beta / 2)}(\mathbf{P}) V_{\mathbf{z}}^{(1+\beta / 2)}}{\lambda_{\max }(\mathbf{P})}-\frac{b}{\lambda_{\max }(\mathbf{P})} V_{\mathbf{z}} .
$$

When $|y|>\varepsilon$, combining with (12) gives

$$
\begin{aligned}
\dot{V}_{m 1}= & \left(|y|^{\beta-1}+1\right) z^{T}\left(\tilde{A}^{T} P+P \tilde{A}\right) z+\dot{m}_{1} \widetilde{m}_{1} \\
= & -a \varphi_{1}(y) \varphi_{2}(y)+(a+c) \varphi_{1}(y) \varphi_{2}(y) \widetilde{m}_{1} \\
= & -c \varphi_{1}(y) \varphi_{2}(y)\left|\widetilde{m}_{1}\right| \\
= & -\sqrt{2} c \varphi_{1}(y) \varphi_{2}(y) V_{k}^{(1 / 2)}, \\
\dot{V}_{2} \leq & -\frac{b \lambda_{\min }^{(1-\beta / 2)}(P) V_{z}^{(1+\beta / 2)}}{\lambda_{\max }(P)}-\frac{b}{\lambda_{\max }(P)} V_{z} \\
& -\sqrt{2} c \varphi_{1}(y) \varphi_{2}(y) V_{k}^{(1 / 2)} \\
= & -\mu V_{2}^{\gamma},
\end{aligned}
$$

with

$$
\begin{aligned}
\mu & =\mu \frac{b \lambda_{\min }^{(1-\beta / 2)}(\mathbf{P}) V_{\mathbf{z}}^{(1+\beta / 2)}}{\lambda_{\max }(\mathbf{P})}, \frac{b}{\lambda_{\max }(\mathbf{P})}, \quad \sqrt{2} c \varphi_{1}(y) \varphi_{2}(y)>0 . \\
\gamma & =\gamma\left(\frac{1+\beta}{2}, 1, \frac{1}{2}\right) .
\end{aligned}
$$

It is known that $(1 / 2)<\gamma<1$ from $(1 / 2) \leq \beta<1$, so

$$
\dot{V}_{2} \leq-\mu V_{2}^{\gamma} \leq 0 .
$$

According to Lemma $1, V_{2}$ can converge in finite time, $y$ can converge to $|y| \leq \varepsilon$, and the convergence time satisfies

$$
t_{1} \leq \frac{V_{2}^{1-\gamma}(0)}{\mu(1-\gamma)} \text {. }
$$

When $|y| \leq \varepsilon$, if $m_{1}$ and $m_{2}$ decrease to the point where the interference cannot be eliminated, the system state will deviate from $|y| \leq \varepsilon$. In this case, $m_{1}$ and $m_{2}$ will increase again under the effect of the adaptive law, making the system state converge to $|y| \leq \varepsilon$.

\section{Guidance Law Design}

The terminal sliding mode control adopts the nonlinear function as the sliding mode surface, which can make the system states converge in finite time, but the method has singular problems. In order to avoid singular problems, based on the piecewise sliding surface [12] and Lemma 3, a nonsingular fast terminal sliding surface is designed as

$$
s=x_{2}+k_{1}\left|x_{1}\right|^{\alpha_{1}} \operatorname{sgn}\left(x_{1}\right)+k_{2} \psi\left(x_{1}\right),
$$

with

$$
\psi\left(x_{1}\right)= \begin{cases}x_{1}^{\alpha_{2}}, & \left|x_{1}\right| \geq \delta, \\ g\left(x_{1}\right), & \left|x_{1}\right|<\delta,\end{cases}
$$

where $\alpha_{1}>1,0<\alpha_{2}=\left(p_{1} / p_{2}\right)<1, k_{1}>0, k_{2}>0, \delta>0$, and $p_{1}$ and $p_{2}$ are positive odd numbers.

Differentiating $s$ with respect to time gives

$$
\dot{s}=\dot{x}_{2}+k_{1} \alpha_{1}\left|x_{1}\right|^{\alpha_{1}-1} x_{2}+k_{2} \psi \prime\left(x_{1}\right) x_{2},
$$

with

$$
\psi^{\prime}\left(x_{1}\right)= \begin{cases}\alpha_{2} x_{1}^{\alpha_{2}-1}, & \left|x_{1}\right| \geq \delta, \\ g^{\prime}\left(x_{1}\right), & \left|x_{1}\right|<\delta,\end{cases}
$$

where $g\left(x_{1}\right)$ is a function of $x_{1}$ and satisfies the following conditions:

(1) $g\left(x_{1}\right)$ is a smooth function in $x_{1} \in(-\delta, \delta)$ with the same sign as $x_{1}$

(2) $g(\delta)=\psi(\delta)=-g(-\delta)$

(3) $g^{\prime}(\delta)=g^{\prime}(-\delta)=\psi^{\prime}(\delta)$, and $\quad g^{\prime}\left(x_{1}\right)>0 \quad$ in $x_{1} \in(-\delta, \delta)$

Remark 4. Condition (1) can ensure that $g^{\prime}\left(x_{1}\right)$ is a continuous bounded function and eliminates singular problems, and when the system reaches the sliding surface $s=0, x_{1}$ and $x_{2}$ are always with different signs, ensuring that the system state is convergent. Condition (2) ensures that the sliding surface $s$ is a continuous function. Condition (3) guarantees that $g\left(x_{1}\right)$ is bounded in $x_{1} \in(-\delta, \delta)$, and $\psi^{\prime}\left(x_{1}\right)$ is a continuous function, so $s$ is a smooth function.

According to the above conditions, this paper selects function $g\left(x_{1}\right)$ as follows:

$$
g\left(x_{1}\right)=\lambda_{1} x_{1}+\lambda_{2} x_{1}^{3},
$$

where $\lambda_{1}=\left(3-\alpha_{2} / 2\right) \varepsilon^{\alpha_{2}-1}$ and $\lambda_{2}=\left(\alpha_{2}-1 / 2\right) \varepsilon^{\alpha_{2}-3}$.

Substituting (3) into (38) yields

$$
\dot{s}=f_{1} x_{2}+f_{2} u+d+k_{1} \alpha_{1}\left|x_{1}\right|^{\alpha_{1}-1} x_{2}+k_{2} \psi^{\prime}\left(x_{1}\right) x_{2} \text {. }
$$

The equivalent guidance law is designed as

$$
u_{\mathrm{eq}}=-f_{2}^{-1}\left(f_{1}+k_{1} \alpha_{1}\left|x_{1}\right|^{\alpha_{1}-1}+k_{2} \psi^{\prime}\right) x_{2} .
$$

Substituting (42) into (41) gives

$$
\dot{s}=d
$$

In order to counteract the disturbance, suppress chattering, and accelerate the convergence speed of the sliding surface, based on the adaptive smooth, fast supertwisting algorithm proposed in the second section, an auxiliary guidance law is designed as 


$$
u_{\mathrm{aux}}=-f_{2}^{-1} k_{3} \varphi_{1}(s)+k_{4} \int_{0}^{t} \varphi_{2}(s) \mathrm{d} t .
$$

The parameter adaptive law is designed as

$$
\left\{\begin{array}{l}
\dot{k}_{3}=(a+c) \varphi_{1}(s) \varphi_{2}(s) \operatorname{sgn}(|s|-\varepsilon), \\
k_{4}=b k_{3} \\
k_{3}(0)>0
\end{array}\right.
$$

Combining with (42) and (44), we can design a nonsingular terminal sliding mode guidance law with the impact angle constraint based on the adaptive supertwisting algorithm as

$$
\begin{aligned}
u= & u_{\mathrm{eq}}+u_{\mathrm{aux}}=-f_{2}^{-1}\left[\left(f_{1}+k_{1} \alpha_{1}\left|x_{1}\right|^{\alpha_{1}-1}+k_{2} \psi^{\prime}\right) x_{2}\right. \\
& \left.+k_{3} \varphi_{1}(s)+k_{4} \int_{0}^{t} \varphi_{2}(s) \mathrm{d} t\right] .
\end{aligned}
$$

For the convenience of description, the design guidance law (46) is abbreviated as ASNTSMG.

\section{Simulation Analysis}

In order to test the performance of the designed guidance law, ASNTSMG, this section conducts simulation analysis based on ballistic simulation in different scenarios. The initial position of the missile is $(0 \mathrm{~m}, 0 \mathrm{~m})$, and the initial position of the target is $(1000 \mathrm{~m}, 5000 \mathrm{~m})$. The missile's velocity is $v_{m}=500 \mathrm{~m} / \mathrm{s}$, and the target's velocity is $v_{t}=250 \mathrm{~m} / \mathrm{s}$. The acceleration of gravity is $g=9.8 \mathrm{~m} / \mathrm{s}^{2}$, and the maximum available overload of the missile is $20 \mathrm{~g}$. The parameters of ASNTSMG are set as follows: $k_{1}=k_{2}=2, a_{1}=3, \alpha_{2}=(5 / 7)$, $\varepsilon=0.01, \delta=0.001, a=0.5$, and $b=c=1$.

In order to verify the superiority of the designed guidance law, this section also carries out the nonsingular fast terminal sliding mode guidance law (NFTSMG) proposed in [23] and the second-order nonsingular terminal sliding mode guidance law (SONTSMG) proposed in [24] to perform a comparative simulation. The expression of NFTSMG is

$$
\begin{aligned}
u= & \frac{r}{\cos \left(q-\theta_{m}\right)}\left[\frac{1}{k_{2} a_{2}} x_{2}^{2-a_{2}}\left(1+k_{1} a_{1} x_{1}^{a_{1}-1}\right)-\frac{2 \dot{r}}{r} x_{2}\right. \\
& \left.+\frac{\alpha s+\beta|s|^{\gamma} \operatorname{sgn}(s)}{r}\right] .
\end{aligned}
$$

The parameters are set as follows: $\alpha=600, \beta=500$, $\alpha_{1}=(7 / 5), \alpha_{2}=(5 / 7), k_{1}=k_{2}=2$, and $\gamma=0.5$.

The expression of SONTSMG is

$$
\begin{aligned}
u= & -\frac{2 \dot{r}}{r} x_{2}+\frac{r \beta}{\alpha} x_{2}^{2-\alpha}+z_{1}+k_{1}|s|^{1-(1 / \gamma)} \operatorname{sgn}(s) \\
& +k_{2} \int_{0}^{t} \frac{x_{2}^{\alpha-1}}{r}|s|^{1-(2 / \gamma)} \operatorname{sgn}(s) \mathrm{d} t .
\end{aligned}
$$

The parameters are set as follows: $k_{1}=600, k_{2}=100$, $\alpha=(7 / 5), \beta=0.5$, and $\gamma=2.1$.
The average overload $N_{\text {me }}$ (unit: g) is introduced to evaluate the energy consumption in the process of guidance, which is defined as follows:

$$
N_{\mathrm{me}}=\frac{1}{K} \sum_{i=1}^{K}\left|a_{m}(i)\right|,
$$

where $K$ is the total number of simulation steps.

Case 1. Attack moving target with different impact angle constraints: set $q_{d}$ as $20^{\circ}, 30^{\circ}, 40^{\circ}$, and $50^{\circ}$, respectively, and $\theta_{m 0}=45^{\circ}$. The target makes sinusoidal maneuver, and its acceleration is $a_{t}=30 \sin (\pi \mathrm{t} / 5) \mathrm{m} / \mathrm{s}^{2}$, and $\theta_{t 0}=150^{\circ}$. The simulation results are shown in Figure 2.

It can be seen from Figures 2(a) and 2(b) that ASNTSMG can effectively intercept the target with different impact angle constraints. The miss distances are $0.374 \mathrm{~m}, 0.428 \mathrm{~m}$, $0.408 \mathrm{~m}$, and $0.479 \mathrm{~m}$, respectively. This method can hit the target accurately. It can be seen from Figures 2(c)-2(e) that the sliding surface and the LOS angular rate can converge to zero in finite time, and LOS angle can effectively converge to the expected impact angle. With the increase of $q_{d}$, the convergence time increases. This is because the larger $q_{d}$ is, the larger the initial LOS angle deviation will be, and the convergence time is related to the initial value, which leads to the corresponding growth of the convergence time.

Figure 2(f) shows the overload curve of the missile, which is saturated in the early stage, and the larger $\theta_{m 0}$, the longer the saturation time, which is mainly due to the larger overload needed in the earlier stage, which makes the missile meet the requirements of angle constraint and guidance accuracy. When $q$ and $\dot{q}$ approach the expected values, the overload gradually approaches zero, which ensures that the missile has sufficient overload margin to deal with other unknown disturbances in the later stage of guidance.

Case 2. Comparative simulation of ASNTSMG, NFTSMG, and SONTSMG: the relevant initial parameters are set to $\theta_{m 0}=45^{\circ}, q_{d}=45^{\circ}$, and $\theta_{t 0}=180^{\circ}$. The movement of the target is set as follows:

(1) Cosine motion: $a_{t}=30 \cos (\pi \mathrm{t} / 5) \mathrm{m} / \mathrm{s}^{2}$

(2) Square wave motion: $a_{t}=30 \operatorname{sgn}(\sin (\pi \mathrm{t} / 5)) \mathrm{m} / \mathrm{s}^{2}$

The simulation results are shown in Figures 3-6 and Table 1.

Figure 3 shows the trajectories of the missile and the target. It can be seen that the missile can track and intercept the target under the three guidance laws. Compared with NFTSMG and SONTSMG, the trajectory of ASNTSMG is relatively smooth, indicating that its attack time is relatively short, which can be verified by Table 1 . Figure 4 shows the LOS angle curve. All three guidance laws can make the LOS angle gradually converge to the expected angle. ASNTSMG can make the LOS angle converge to the expected angle more quickly. NFTSMG adopts the robustness of the sliding mode control to cancel the disturbance of target maneuver, so it can only make the system states converge to the neighborhood of origin in finite time. NFTSMG and SONTSMG adopt the traditional nonsingular terminal sliding surface, 

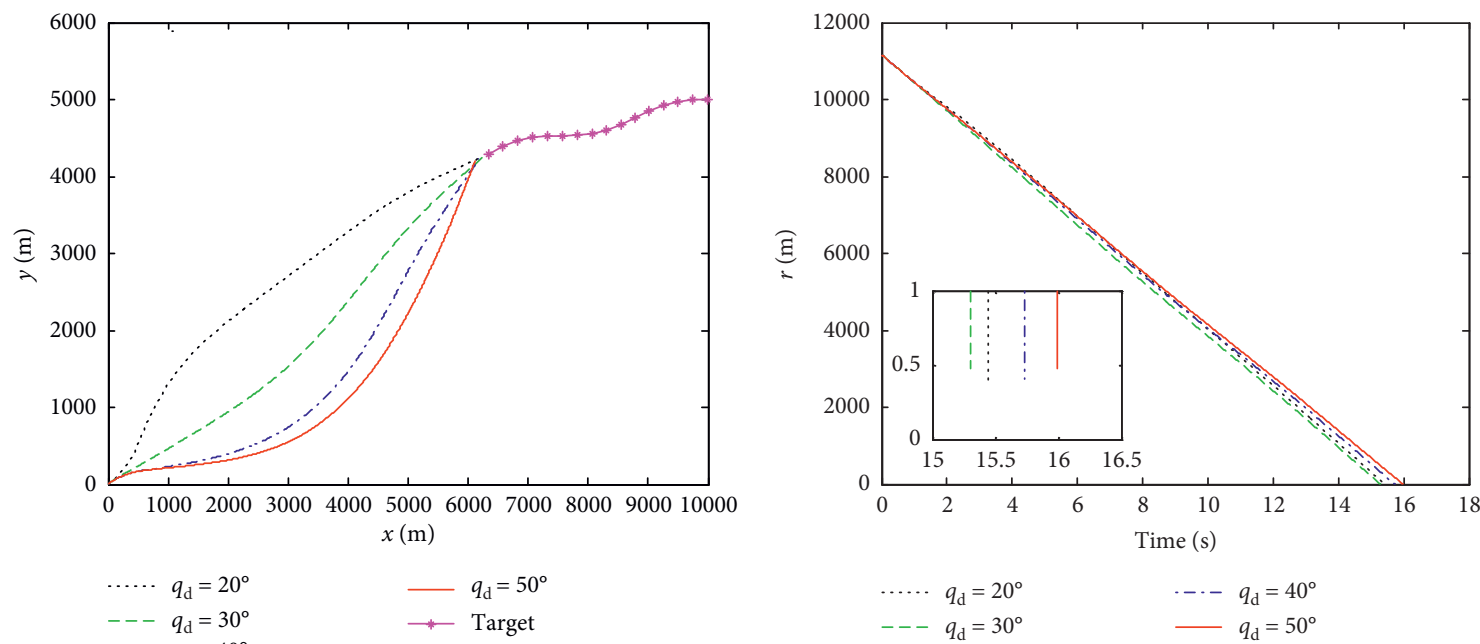

(a)

(b)

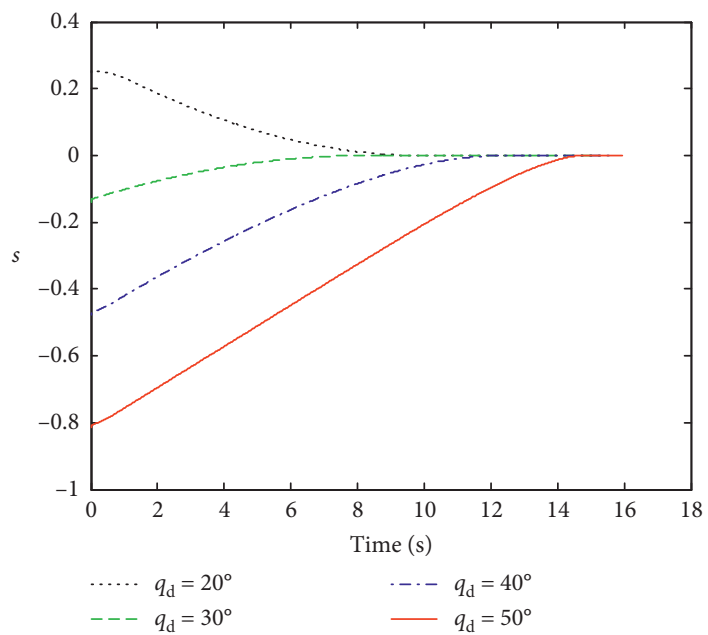

(c)

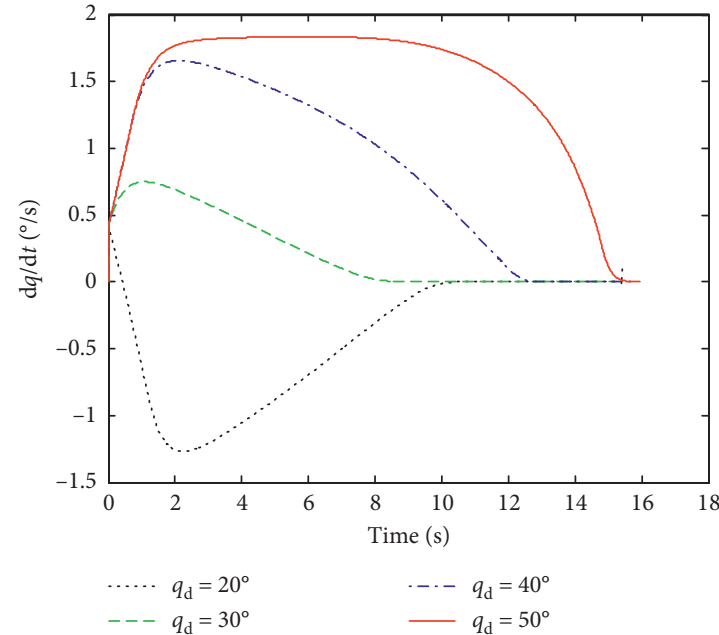

(e)

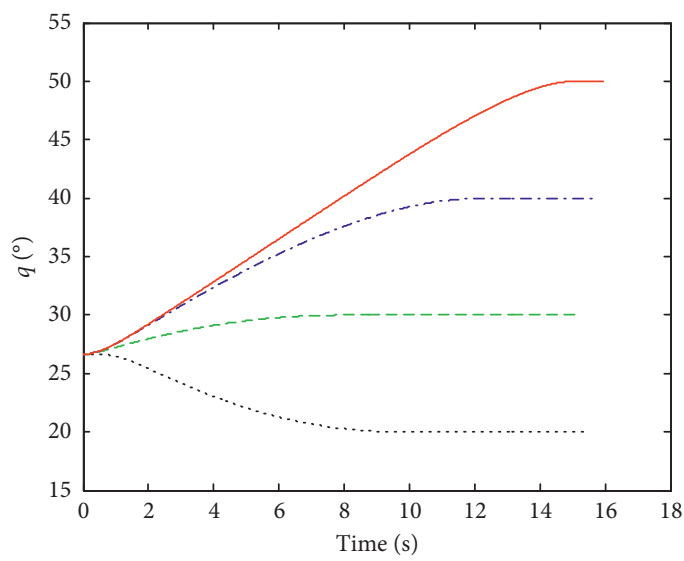

(d)

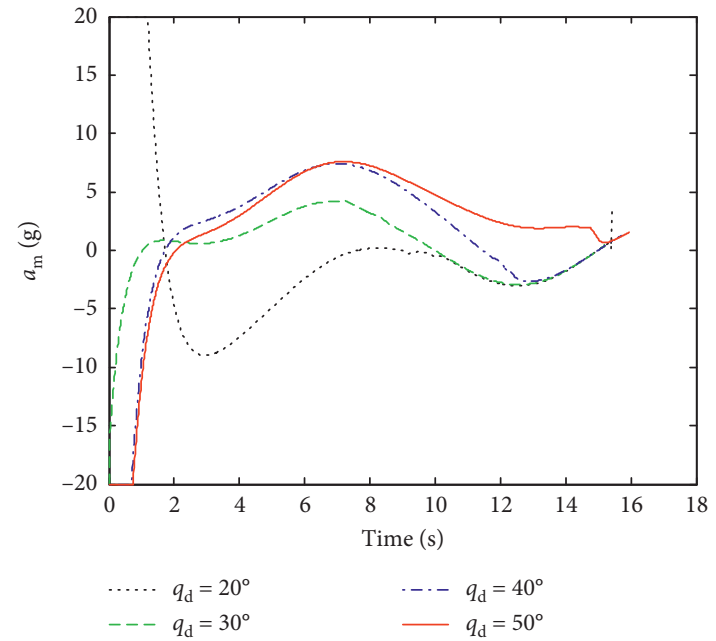

(f)

Figure 2: Simulation results of Case 1. (a) Missile and target motion trajectory. (b) Relative distance between the missile and the target. (c) Sliding mode. (d) LOS angle. (e) LOS angular rate. (f) Missile overload. 


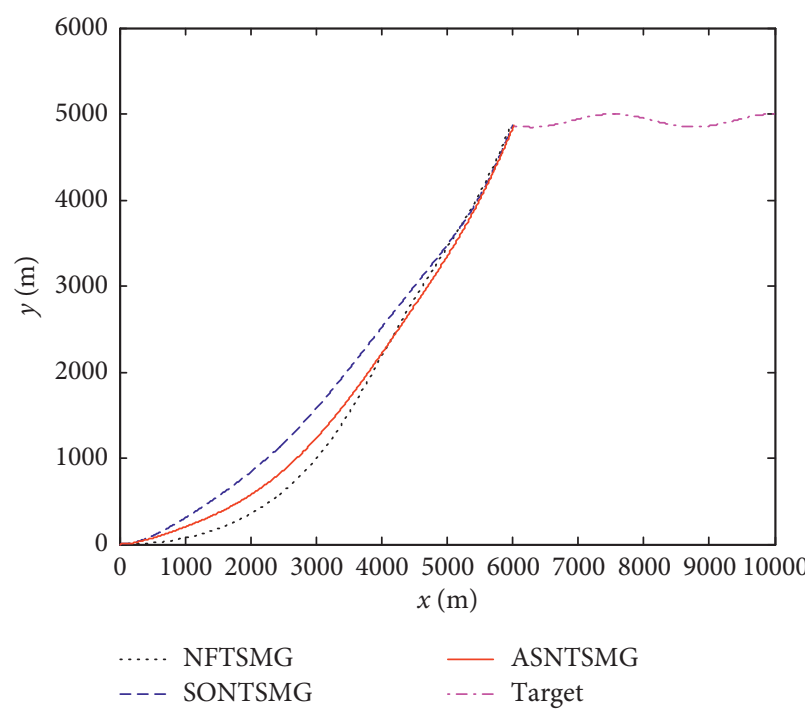

(a)

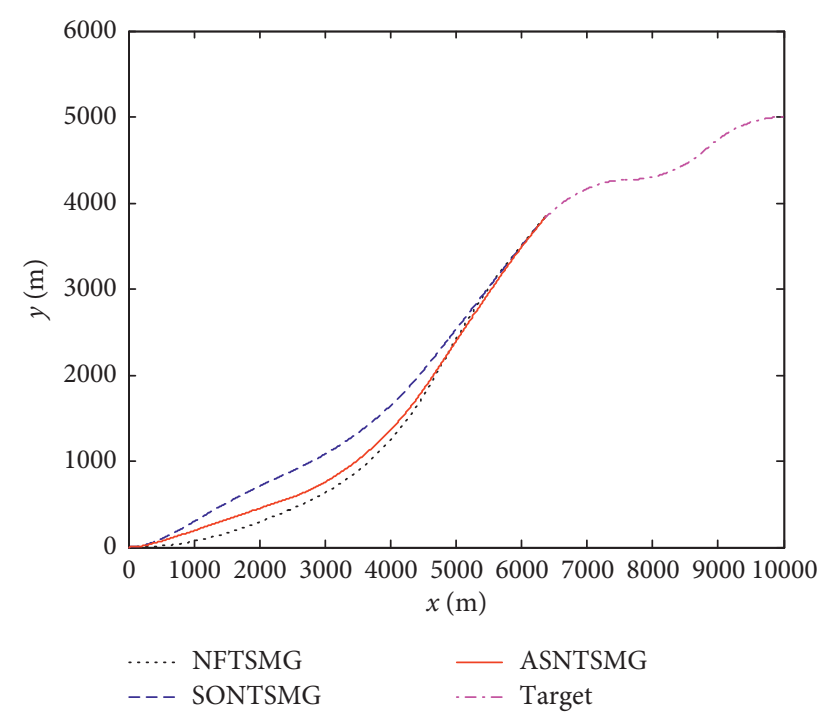

(b)

Figure 3: Missile and target motion trajectory. (a) Target cosine motion. (b) Target square wave motion.

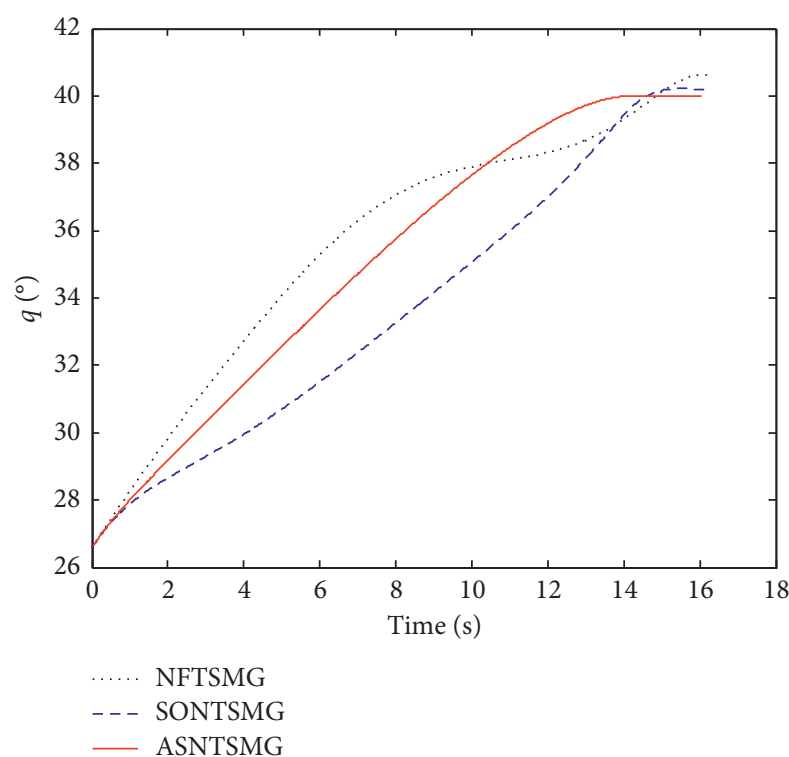

(a)

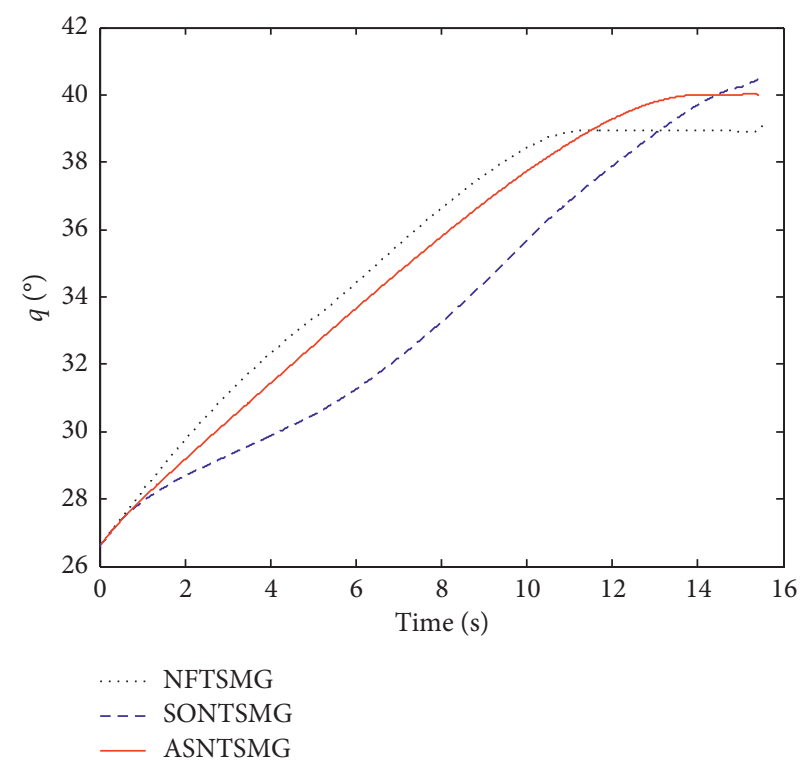

(b)

FIgURE 4: LOS angle. (a) Target cosine motion. (b) Target square wave motion.

which has the nonstrict finite-time convergence problem, and the nonconvergence factor slows down the convergence speed. Figure 5 shows the LOS angular rate curve. Under the three guidance laws, the LOS angular rates converge to zero in finite time. ASNTSMG has smaller convergence error and faster convergence speed. Figure 6 shows the overload curve of the missile. Due to the finite-time convergence of LOS angle and angular rate, the missile needs a large overload in the early stage of guidance. Therefore, the overloads of three guidance laws are all saturated in the early stage. When the LOS angle and the angular rate converge, the missile overload gradually converges to zero in the later stage. And the convergence speed of ASNTSMG is faster.

Table 1 shows the simulation results of attack time, miss distance, LOS angle error, and average overload under the three guidance laws. It can be seen that compared with NFTSMG and SONTSMG, ASNTSMG has smaller attack time, miss distance, terminal LOS angle error, and average overload, so ASNTSMG has better guidance performance.

According to the analysis of the simulation results of two cases, ASNTSMG can hit the target precisely with the 


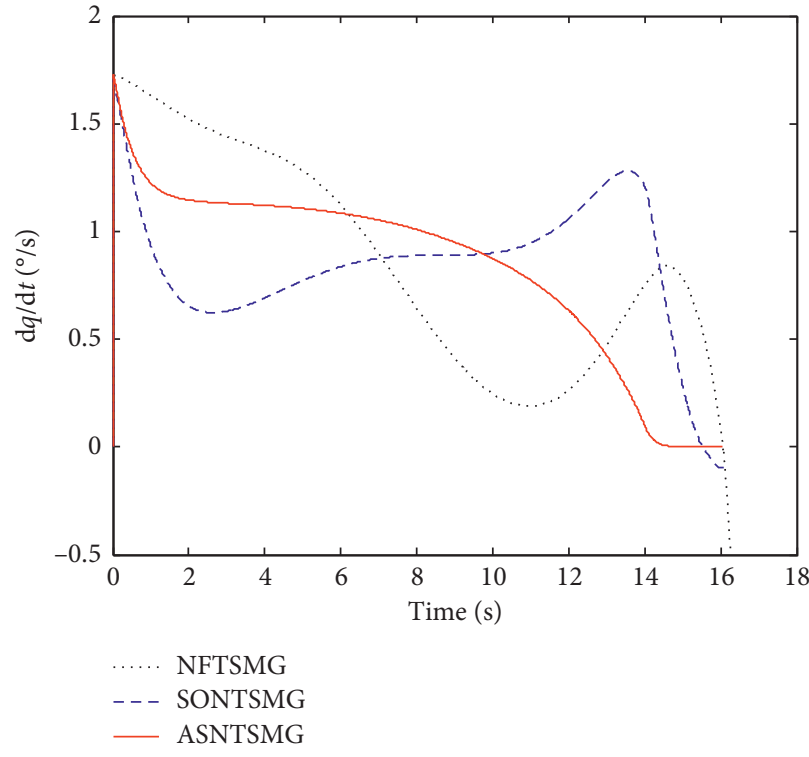

(a)

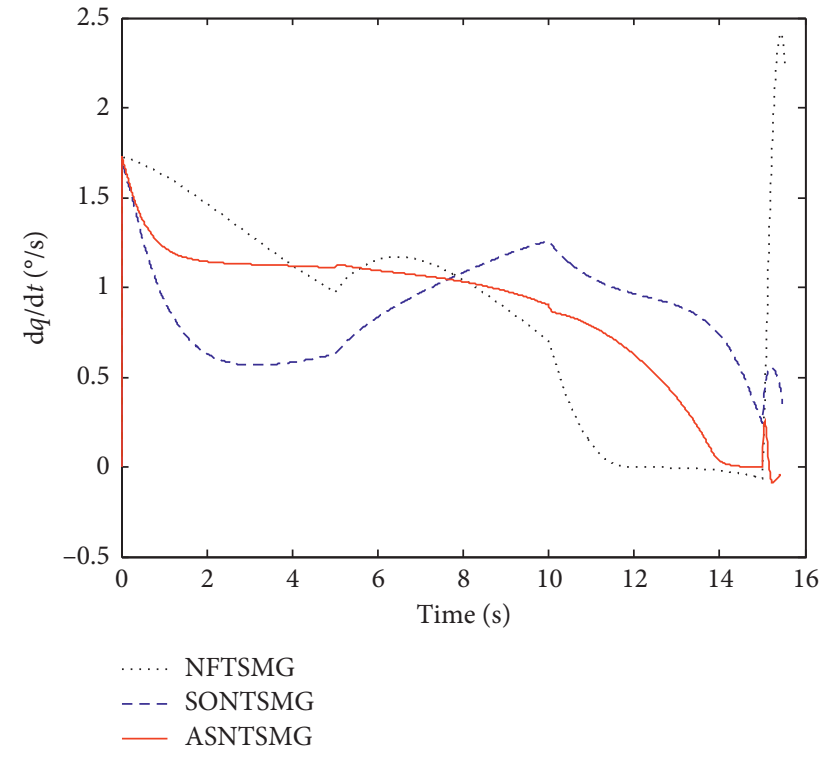

(b)

FIgURE 5: LOS angular rate. (a) Target cosine motion. (b) Target square wave motion.

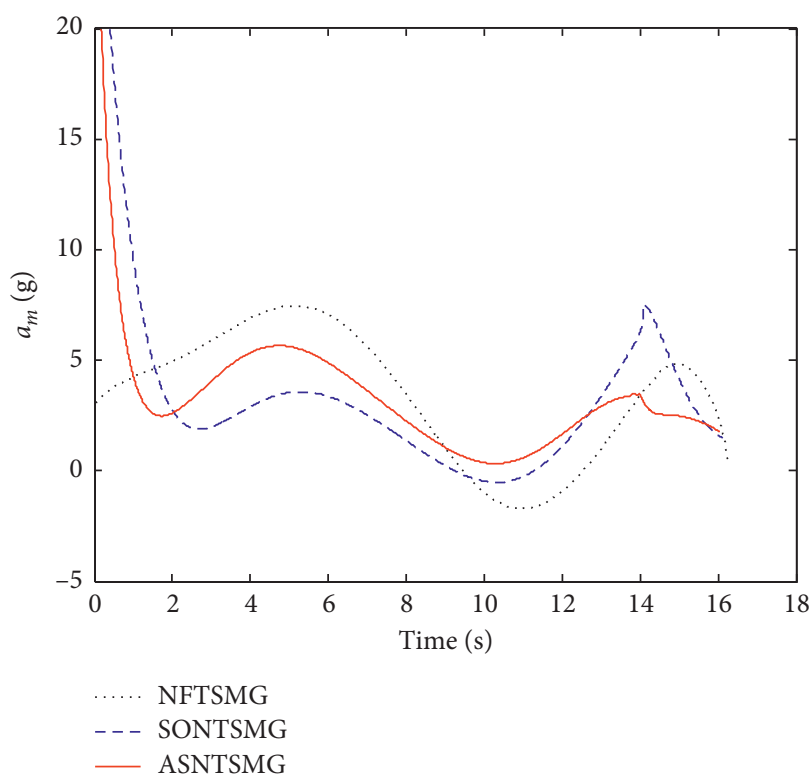

(a)

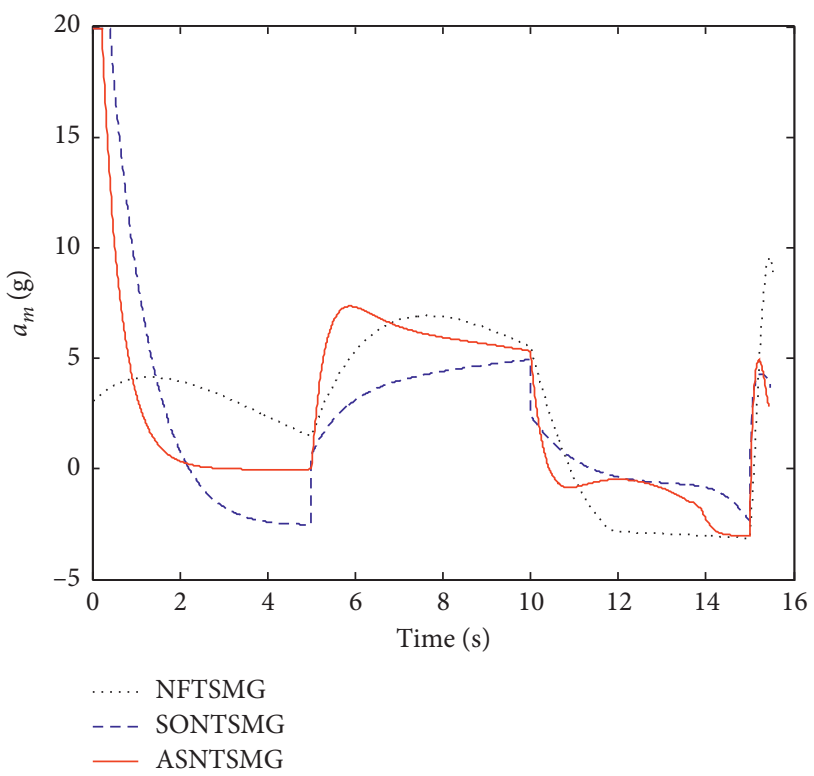

(b)

Figure 6: Missile overload. (a) Target cosine. (b) Target square wave motion.

TABLE 1: Simulation results of different guidance laws.

\begin{tabular}{|c|c|c|c|c|c|}
\hline Target movement & Guidance law & Attack time (s) & Miss distance $(\mathrm{m})$ & Angle error (deg) & $N_{\text {me }}(\mathrm{g})$ \\
\hline \multirow{3}{*}{ Cosine maneuver } & NFTSMG & 16.24 & 0.95 & 0.59 & 3.88 \\
\hline & SONTSMG & 16.12 & 0.76 & 0.21 & 3.58 \\
\hline & ASNTSMG & 16.05 & 0.39 & 0.02 & 3.43 \\
\hline \multirow{3}{*}{ Square wave maneuver } & NFTSMG & 15.54 & 1.03 & 0.88 & 3.99 \\
\hline & SONTSMG & 15.47 & 0.86 & 0.58 & 3.48 \\
\hline & ASNTSMG & 15.41 & 0.43 & 0.03 & 3.36 \\
\hline
\end{tabular}


expected impact angle under different expected LOS angles and target maneuvering conditions. Compared with the existing guidance laws NFTSMG and SONTSMG, ASNTSMG can effectively attack the target with less impact angle error, miss distance, and energy consumption, which verifies the effectiveness and superiority of ASNTSMG.

\section{Conclusion}

In this paper, a nonsingular fast terminal sliding mode guidance law is proposed to solve the problem of guidance accuracy and impact angle constraint. Through theoretical analysis and simulation verification, the following conclusions can be obtained:

(1) The proposed adaptive smooth supertwisting algorithm can effectively counteract the disturbance of the system and accelerate the convergence speed of the system without knowing the upper bound of the disturbance.

(2) The designed nonsingular terminal sliding mode surface can realize the fast finite-time convergence of the system states and ensure the impact angle constraint and guidance accuracy requirements.

(3) This guidance law can attack the target precisely under the conditions of different expected LOS angles and target maneuvers. Compared with the existing nonsingular fast terminal sliding mode guidance law and second-order nonsingular terminal sliding mode guidance law, this law has higher guidance accuracy and angle constraint accuracy and consumes less energy.

\section{Data Availability}

The data used to support the findings of this study are included within the article.

\section{Conflicts of Interest}

The authors declare that there are no conflicts of interest regarding the publication of this paper.

\section{References}

[1] H. Cai, Z. D. Hu, and Y. Cao, "A survey of guidance law with terminal impact angle constraints," Journal of Astronautics, vol. 31, no. 2, pp. 315-323, 2010.

[2] J. M. Song and T. Q. Zhang, "Passive homing missile's variable structure proportional navigation with terminal angular constraint," Chinese Journal of Aeronautics, vol. 14, no. 2, pp. 83-87, 2001.

[3] P. Wu and M. Yang, "Variable structure guidance law with terminal attack angle constraint," Journal of Solid Rocket Technology, vol. 31, no. 2, pp. 116-120, 2002.

[4] S. R. Kumar, S. Rao, and D. Ghose, "Sliding-mode guidance and control for all-aspect interceptors with terminal angle constraints," Journal of Guidance, Control, and Dynamics, vol. 35, no. 4, pp. 1230-1246, 2012.

[5] H. B. Zhou, S. M. Song, and M. Y. Xu, "Design of terminal sliding-mode guidance law with attack angle constraints," in
Proceedings of the 2013 25th Chinese Control and Decision Conference (CCDC), Guiyang, China, May 2013.

[6] Y. X. Zhang, M. W. Sun, and Z. Q. Chen, "Finite-time convergent guidance law with impact angle constraint based on sliding-mode control," Nonlinear Dynamic, vol. 7, no. 3, pp. 619-625, 2012.

[7] X. H. Yu and Z. H. Man, "Fast terminal sliding-mode control design for nonlinear dynamical systems," IEEE Transactions on Circuits and Systems I: Fundamental Theory and Applications, vol. 49, no. 2, pp. 261-264, 2002.

[8] J. M. Song, S. M. Song, and Y. Guo, "Nonlinear disturbance observer based fast terminal sliding mode guidance with impact angle constraints," International Journal of Innovative Computing, Information and Control, vol. 11, no. 3, pp. 787-802, 2015.

[9] S. R. Kumar, S. Rao, and D. Ghose, "Nonsingular terminal sliding mode guidance with impact angle constraints," Journal of Guidance, Control, and Dynamics, vol. 37, no. 4, pp. 1114-1130, 2014.

[10] S. F. Xiong, W. H. Wang, and S. Wang, "Nonsingular fast terminal sliding-mode guidance with intercept angle constraint," Control Theory \& Applications, vol. 31, no. 3, pp. 269-278, 2014.

[11] S. C. Yang, K. Q. Zhang, and P. Chen, "Adaptive terminal sliding mode guidance law with impact angle constraint," Journal of Beijing University of Aeronautics and Astronautics, vol. 42, no. 8, pp. 1566-1574, 2016.

[12] Z. L. Zhang and J. Zhou, "Strictly convergent non-singular terminal sliding mode guidance law with impact angle constraints," Optik, vol. 127, no. 22, pp. 10971-10980, 2016.

[13] Z. Zhang, C. Man, S. Li, and S. Jin, "Finite-time guidance laws for three-dimensional missile-target interception," Journal of Aerospace Engineering, vol. 230, no. 2, pp. 392-403, 2015.

[14] N. Zhang, W. Gai, M. Zhong, and J. Zhang, "A fast finite-time convergent guidance law with nonlinear disturbance observer for unmanned aerial vehicles collision avoidance," Aerospace Science and Technology, vol. 86, pp. 204-214, 2019.

[15] J. Song, S. Song, and H. Zhou, "Adaptive nonsingular fast terminal sliding mode guidance law with impact angle constraints," International Journal of Control, Automation and Systems, vol. 14, no. 1, pp. 99-114, 2016.

[16] J. Song and S. Song, "Robust impact angle constraints guidance law with autopilot lag and acceleration saturation consideration," Transactions of the Institute of Measurement and Control, vol. 41, no. 1, pp. 182-192, 2019.

[17] A. Levant, "Principles of 2-sliding mode design," Automatica, vol. 43, no. 4, pp. 576-586, 2007.

[18] A. M. Zhou, "Finite-time output feedback attitude tracking control for rigid spacecraft," IEEE Transactions on Control Systems Technology, vol. 22, no. 1, pp. 338-345, 2014.

[19] B. Li, Q. Hu, Y. Yu, and G. Ma, “Observer-based fault-tolerant attitude control for rigid spacecraft," IEEE Transactions on Aerospace and Electronic Systems, vol. 53, no. 5, pp. 25722582, 2017.

[20] H. Li and Y. Cai, "On SFTSM control with fixed-time convergence," IET Control Theory \& Applications, vol. 11, no. 6, pp. 766-773, 2017.

[21] J. Ni, L. Liu, C. Liu, X. Hu, and T. Shen, "Fixed-time dynamic surface high-order sliding mode control for chaotic oscillation in power system," Nonlinear Dynamics, vol. 86, no. 1, pp. 401-420, 2016.

[22] Q. Zong, Z. S. Zhao, and J. Zhang, "Higher order sliding mode control with self-tuning law based on integral sliding mode," 
IET Control Theory and Application, vol. 4, no. 7, pp. 12821289, 2008.

[23] S. He, D. Lin, and J. Wang, "Continuous second-order sliding mode based impact angle guidance law," Aerospace Science and Technology, vol. 41, pp. 199-208, 2015.

[24] B. L. Cong, Z. Chen, and X. D. Liu, "On adaptive sliding mode control without switching gain overestimation," International Journal of Robust and Nonlinear Control, vol. 24, no. 3, pp. 515-531, 2014. 